Proceedings

\title{
Beetroot as a Source of Natural Dyes for Ham ${ }^{+}$
}

\author{
Sandra Dias ${ }^{1}$, David M. Pereira ${ }^{2}$, Elisabete M. S. Castanheira ${ }^{3}$, A. Gil Fortes ${ }^{1}$, Regina Pereira ${ }^{4}$, \\ and M. Sameiro T. Gonçalves 1,* \\ 1 Centre of Chemistry (CQ/UM), University of Minho, Campus of Gualtar, 4710-057 Braga, Portugal; \\ sandraisdias@hotmail.com (S.D.); gilf@quimica.uminho.pt (A.G.F.) \\ 2 REQUIMTE/LAQV, Laboratory of Pharmacognosy, Department of Chemistry, Faculty of Pharmacy, \\ University of Porto, R. Jorge Viterbo Ferreira, 228, 4050-313 Porto, Portugal; dpereira@ff.up.pt \\ 3 Centre of Physics (CFUM), University of Minho, Campus de Gualtar, 4710-057 Braga, Portugal; \\ ecoutinho@fisica.uminho.pt \\ 4 Primor Charcutaria-Prima, S.A, Avenida Santiago de Gavião, no 1142 Gavião, \\ 4760-003 Vila Nova de Famalicão, Portugal; regina.pereira@primor.pt \\ * Correspondence: msameiro@quimica.uminho.pt \\ + Presented at the 23rd International Electronic Conference on Synthetic Organic Chemistry, 15 November- \\ 15 December 2019; Available online: https://ecsoc-23.sciforum.net/.
}

Published: 14 November 2019

\begin{abstract}
Beetroot (Beta vulgaris L.) was subjected to extraction procedures in order to obtain the respective extracts containing the natural dyes and subjected to cytotoxicity assays in AGS cell line. Encapsulation of the extracts in nanosystems based on soybean lecithin and maltodextrin was performed. Lyophilized extracts before and after encapsulation in maltodextrin were applied in the formulation of leg ham and used in pilot scale of production. The colour of ham samples from the different assays was evaluated visually and by colorimetry.
\end{abstract}

Keywords: ham; beetroot; Beta vulgaris L.; natural dyes; betalain pigments; betacyanins

\section{Introduction}

Due to the composition and processing of leg ham, in some cases the final colour is not the most attractive. Legislation does not allow the use of synthetic dyes nor some natural dyes, such as carmine dye (E120) from the cochineal insect, capable of conferring the pink/carmine colour, commercially available and presently used in other food products.

Beetroot (Beta vulgaris L.) is a root vegetable possessing a variety of components with numerous nutritional and health benefits, including water-soluble betalain pigments like betacyanins (redviolet colour) and betaxanthins (yellow-orange colour) [1-3]. The use of beetroot as an ingredient in several products imparts beneficial effects on human health and provides an opportunity for development of different functional foods [4-6].

Considering these facts, Beta vulgaris L. was selected as a source of natural pigments, in order to obtain betalain-rich extracts, which were partially characterized by analytical techniques, and subjected to cytotoxicity studies in the AGS cell line. The extracts were used in nanoencapsulation studies on soybean lecithin and maltodextrin. With the aim of evaluating the colouration capability in ham of lyophilized extracts before and after nanoencapsulation in maltodextrin, they were included in appropriated formulations and used in a leg ham pilot scale of production. Visual observation and colorimetry measurements were carried out with coloured samples obtained in comparison of non-coloured ham. 


\section{Materials and Methods}

\subsection{Preparation of Betalain-Rich Extract}

The red beetroot (Beta vulgaris L.) used was obtained from Portugal in October 2019. A solution of water: ethanol: acetic acid (66.6:33:0.33, $v / v / v)(100 \mathrm{~mL})$ was added to the plant material (previously hand-peeled and cut into small pieces $(50 \mathrm{~g})$ ), and the mixture was kept at room temperature for 48 h. After this time, the mixture was filtered, and then partially evaporated in the rotary evaporator at $40{ }^{\circ} \mathrm{C}$. Then, the extract was frozen at $-80^{\circ} \mathrm{C}$ and lyophilized (Alpha 1-4 LD Plus-Crist freeze dryer) for five days, resulting in the corresponding dry extract that was stored in a desiccator until use.

\subsection{Determination of Betalain Content}

Absorbance values of the extract were measured in a Shimadzu UV-3600 Plus UV-vis-NIR spectrophotometer (Shimadzu Corporation, Kyoto, Japan) at $538 \mathrm{~nm}$ and $480 \mathrm{~nm}$ against a blank of ultrapure water. These values were then used to calculate the betacyanins and betaxanthins content, reported as mg equivalent betanin/L and $\mathrm{mg}$ equivalent indicaxanthin/L, respectively. Betacyanins were detected at $538 \mathrm{~nm}$ and betaxanthins at $480 \mathrm{~nm}$, according to Equation (1):

$$
\text { betacyanins or betaxanthins content }[\mathrm{mg} / \mathrm{L}]=[(\mathrm{A} \times \mathrm{DF} \times \mathrm{MW} \times 1000 / \varepsilon \times l)] \text {, }
$$

where $\mathrm{A}$ is the absorbance at 538 or $480 \mathrm{~nm}$, DF is the dilution factor, MW is the molecular weight, $\varepsilon$ is the absorption coefficient and $l$ is the optical path $(1 \mathrm{~cm})$. For betacyanin, the molar absorption coefficient is $6.0 \times 10^{4} \mathrm{M}^{-1} \mathrm{~cm}^{-1}$ and $\mathrm{MW}=550 \mathrm{~g} / \mathrm{mol}$. For betaxanthins, the molar absorption coefficient is $4.8 \times 10^{4} \mathrm{M}^{-1} \mathrm{~cm}^{-1}$ and $\mathrm{MW}=308 \mathrm{~g} / \mathrm{mol}[7,8]$.

\subsection{HPLC-DAD Analysis}

The lyophilized extract was re-dissolved in HPLC-grade water acidified with formic acid ( $\mathrm{pH}$ $2.0)$ and filtered by a $0.22 \mu \mathrm{m}$ size pore membrane. A sample $(20 \mu \mathrm{L})$ was injected into a Spherisorb ODS2 column with a C18 ODS guard column at a flow rate of $0.8 \mathrm{~mL} / \mathrm{min}$. The mobile phase consisted of water: formic acid: acetonitrile $(87: 10: 3, v / v / v$; eluent $\mathrm{A})$ and water: formic acid: acetonitrile (40:10:50, v/v/v; eluent B), using a gradient as follows: start with $10 \%$ to obtain $25 \%$ B after $10 \mathrm{~min}$., $31 \% \mathrm{~B}$ at $15 \mathrm{~min} ., 40 \% \mathrm{~B}$ at $20 \mathrm{~min}$., reaching $50 \% \mathrm{~B}$ at $30 \mathrm{~min}$. Chromatograms were recorded at 280, 320,350 and $525 \mathrm{~nm}$.

Betalaines were identified by comparing their retention times and UV-vis spectra (200-600 nm) with those of reference substances and with literature data [3].

\subsection{AGS Cell Assays}

As a model for gastric cells, the human gastric adenocarcinoma cell line AGS was used. Cells were purchased from Sigma-Aldrich (St. Louis, MO, USA) and maintained in medium DMEM + GlutaMAxTM-1 with $1 \%$ penicillin/streptomycin and $10 \% \mathrm{FBS}$, at $37^{\circ} \mathrm{C}$, in a humidified atmosphere of $5 \% \mathrm{CO}_{2}$.

For the assessment of viability, cells were plated at a density of $1.5 \times 10^{4}$ cells/well, followed by incubation for $24 \mathrm{~h}$ with the samples. After an incubation, $0.5 \mathrm{mg} / \mathrm{mL}$ MTT solution was added and further incubated for $2 \mathrm{~h}$. The formazan in each well was dissolved in a solution of DMSO: isopropanol (3:1). Lastly, the absorbance at $560 \mathrm{~nm}$ was read in a Thermo Scientific ${ }^{\mathrm{TM}}$ Multiskan $^{\mathrm{TM}}$ GO microplate reader.

\subsection{Nanoencapsulation Studies}

For nanoencapsulation studies, the lyophilized extract of beetroot was used. Concentration dilutions of $2 \times 10^{-5}-50 \times 10^{-4} \mathrm{mg} / \mathrm{mL}$ were performed to determine the calibration curve and calculate the encapsulation efficiency.

Liposomes were prepared by the ethanolic injection and thin film hydration methods using a commercial lipid mixture for food industry, soy lecithin (Sternchemie, Hamburg, Germany), 
containing ( $\% \mathrm{~mol} / \mathrm{mol}) \quad 22 \%$ phosphatidylcholine, 20\% phosphatidylethanolamine, $20 \%$ phosphatidylinositol, and $10 \%$ acid phosphatidic acid as main components, with a concentration of $1 \times 10^{-3} \mathrm{M}$. In the ethanolic injection method, $2 \times 10^{-3} \mathrm{~g} / \mathrm{mL}$ extract was added to the lipid mixture and the solvent was evaporated with an ultrapure nitrogen stream. After evaporation, tetrahydrofuran $(0.75 \mu \mathrm{L})$ and ethanol $(0.75 \mu \mathrm{L})$ were added. Under vortexing, the mixture was added to ultrapure water $(3 \mathrm{~mL})$, and the resulting solution was placed in Amicon tubes and centrifuged (Universal 320 Hettich Zentrifugen, New Delhi, India), for $10 \mathrm{~min}$ at $3000 \mathrm{rpm}$.

For lipid thin film hydration, soybean lecithin $\left(60 \times 10^{-3} \mathrm{~g}\right)$ was added to ethanol $(3 \mathrm{~mL})$, and the mixture was evaporated under a stream of ultrapure nitrogen. To the lipid mixture, $10 \times 10^{-3} \mathrm{~g} / \mathrm{mL}$ of the aqueous extract solution, previously dissolved in $5 \mathrm{~mL}$ of ultrapure water, was added. This solution was vortexed for $5 \mathrm{~min}$. and sonicated also for $5 \mathrm{~min}$. Then, the resulting mixture was centrifuged in Amicon filter tubes for $10 \mathrm{~min}$ at $3000 \mathrm{rpm}$.

Both the encapsulated and non-encapsulated fractions were collected and absorption spectra were recorded in a spectrophotometer Shimadzu UV-3600 Plus UV-vis-NIR spectrophotometer (Shimadzu Corporation, Kyoto, Japan). The absorbance of the fractions was measured and the concentrations of the encapsulated and non-encapsulated pigments were determined using the calibration curve (absorbance vs. concentration). The encapsulation efficiency, EE(\%), was obtained through Equation (2).

$$
\text { EE }(\%)=(\text { Total amount }- \text { Amount of nonencapsulated extract }) /(\text { Total amount }) \times 100
$$

For nanoencapsulation studies using maltodextrin (DE20) the lyophilized extract (0.5 g) was added to distilled water $(3 \mathrm{~mL})$ and then, under stirring, maltodextrin $(3 \mathrm{~g})$ was added. The resulting mixture was centrifuged (Hemle Z-300) at $500 \mathrm{rpm}$ for $16 \mathrm{~min}$. and then subjected to lyophilization and, based on the final mass obtained, the encapsulation yield, EY (\%), was calculated using Equation (3).

$$
\text { EY }(\%)=(\text { final mass after lyophilization }) /(\text { initial mass }(\text { maltodextrin }+ \text { extract })) \times 100
$$

\subsection{Application of the Beetroot Pigments to Ham}

The ingredients that make up the brine and the different meats to incorporate the ham were weighed. Brine was prepared and the chosen extract was added. The temperature and $\mathrm{pH}$ of the resulting mixture were measured. The meat was later added, and with the help of a food processor (Yammi), the mixture was wrapped to make it homogeneous. The resulting mixture was then packaged in plastic casing and formed into metal containers and kept for $12 \mathrm{~h}$ at $4{ }^{\circ} \mathrm{C}$.

After resting, the paste was cooked at $75^{\circ} \mathrm{C}$, which is complete when the core reaches $72{ }^{\circ} \mathrm{C}$. At this point, the ham was quickly placed on ice to rapidly reach $4{ }^{\circ} \mathrm{C}$ and stored at low temperatures (5 ${ }^{\circ} \mathrm{C}$ ) for $24 \mathrm{~h}$. At the end of this period, the ham was deformed and placed in the cold at $4{ }^{\circ} \mathrm{C}$ until it was used for colorimetric assays.

\subsection{Colour Measurement}

The colour of ham samples was analysed using the CIELab system. The colour parameter values, namely $\mathrm{L}^{*}$ (brightness), $\mathrm{a}^{*}$ (green versus red coordinate) and $\mathrm{b}^{*}$ (blue versus yellow coordinate) were determined on a white calibration block using a Konica Minolta Chroma Meter CR-400/410.

The ham samples, with and without beetroot extracts in the formulation used in their preparation, were cut into slices of about $1.5 \mathrm{~mm}$. The colour measurements were made in a transparent Petri dish $\left(5 \mathrm{~cm}^{2}\right)$, where the ham slices were placed to be analysed. Each sample was analysed at five distinct points.

\section{Results and Discussion}

In order to obtain betalain-rich extracts from beetroot, different experimental conditions, including various solvent systems, were used, revealing that a solution of water, ethanol and acetic acid $(66.6: 33: 0.33, v / v / v)$, at room temperature, with irradiation protection for $48 \mathrm{~h}$, resulted in high 
absorbance liquid extract at about 538 and $480 \mathrm{~nm}$, suggesting significant concentrations of betalain pigments. Consequently, this extract was subjected to lyophilization and partially characterized by different analytical techniques, namely UV-vis absorption spectroscopy, HPLC-DAD, and lowresolution mass spectrometry.

The UV-vis absorption spectrum of the extract showed a broad band with absorption maxima at $537 \mathrm{~nm}$ and $480 \mathrm{~nm}$, which suggests the presence of betaxanthines and betacyanins, respectively (Figure 1). Thus, considering the UV-vis data and applying Equation (1), the betalains content was estimated, resulting in $22.13 \mathrm{mg} / \mathrm{L}$ of betacyanins and $9.63 \mathrm{mg} / \mathrm{L}$ of betaxanthins.

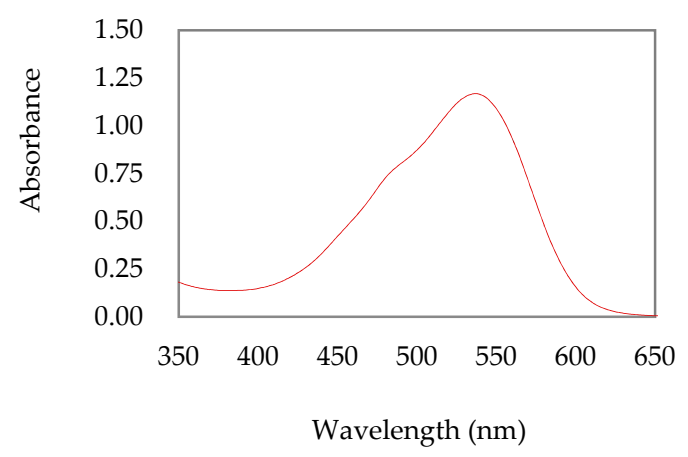

Figure 1. Absorption spectrum of beetroot lyophilized extract in aqueous solution.

The HPLC-DAD studies of the beetroot extract confirmed the presence of betalains, and lowresolution mass spectrometry acquired in positive ionization mode (ESI) displayed a peak with $\mathrm{m} / \mathrm{z}$ 550.78 (base peak) attributed to betanine according to the literature related to beetroot pigment components [3].

Given the specific ham application intended for this work, as well as other potential food applications, the cytotoxicity of lyophilized extract in human gastric adenocarcinoma (AGS) cells was evaluated as a model for gastric cells. The results confirmed the absence of cytotoxicity up to a concentration of $1 \mathrm{mg} / \mathrm{mL}$, as expected.

The ham preparation process involves mixing different additives and treatments with temperature and acidic $\mathrm{pH}$, which can cause structural changes in the pigments used and consequently in the color obtained with them. Given that betalains are sensitive to certain conditions ( $\mathrm{pH}$, temperature and light, among others), and considering their use in coloration of the ham, it was decided to nanoencapsulate the beetroot extract. In this sense, liposomes of soybean lecithin and maltodextrin (DE20) matrices were used, as these systems are reported for nanoencapsulation stabilization of different components, as well as additives in the food industry.

For soybean lecithin, ethanolic injection and thin film hydration methods were used, as they are usually associated with high encapsulation efficiencies $[9,10]$. The results obtained showed high encapsulation efficiencies without a substantial difference between the two procedures $(84.3 \pm 3 \%$ and $94.5 \pm 4 \%$, respectively). However, since the industrial scale application of these processes has both financial and experimental limitations, the maltodextrin (DE20) encapsulation was also carried out, which is easier to perform and economically favorable. The results showed a $100 \%$ yield of nanoencapsulation.

The main objective of this work was to evaluate the possibility of applying natural dyes as an alternative to cochineal carmine (E120) and other dyes not allowed for use with ham according to legislation in order to obtain an attractive pink color for the consumer. Thus, extracts of beetroot nonencapsulated and encapsulated in maltodextin after lyophilization were added to the formulation, and the corresponding leg ham preparations were made on a pilot scale at Primor. In order to evaluate the effect on the color of the pigments present in the extracts, the same type of ham commonly produced and marketed by the company was also prepared. 
Visual evaluation of the ham samples together with the values of color parameters obtained in the colorimeter (Table 1) suggest that the colors obtained with extracts in both cases meet the desired one, being necessary to perform more tests with smaller amounts of non-encapsulated extract in order to verify the minimum quantity required to obtain the same color. In addition, it is necessary to confirm the relevance of encapsulation to stabilize the extract during the ham manufacturing process.

Table 1. Colorimetric data of ham samples prepared without and with beetroot extracts (non)encapsulated.

\begin{tabular}{cccccc}
\hline \multirow{2}{*}{ Sample } & \multicolumn{5}{c}{ Color Parameters } \\
\cline { 2 - 6 } & $\mathbf{L}^{*}$ & $\mathbf{a}^{*}$ & $\mathbf{b}^{*}$ & $\mathbf{C}^{*}$ & $\mathbf{h}$ \\
\hline Ham without extract & 60.74 & -0.32 & 6.84 & 6.85 & 92.92 \\
Ham with extract $(4.55 \mathrm{~g} / \mathrm{kg})$ & 58.37 & 4.64 & 9.64 & 10.69 & 64.12 \\
Ham with encapsulated extract $(7.29 \mathrm{~g} / \mathrm{kg})^{1}$ & 61.54 & 0.36 & 8.71 & 8.73 & 87.84 \\
\hline
\end{tabular}

${ }_{1}^{1}$ Mass of the encapsulated extract corresponds to the ratio $1 \mathrm{~g}$ of non-encapsulated extract $/ \mathrm{kg}$.

Overall, the results obtained in this work suggest that leg ham coloration achieved with lyophilized extracts of Beta vulgaris L. are very promising for future applications in this type of processed meat products.

Funding: This research was funded by Foundation for Science and Technology (FCT) for financial support to the research centres CQ/UM (UID/QUI/00686/2016 and UID/QUI/00686/2019) and CF-UM-UP (UID/FIS/04650/2019). Funding by FCT, COMPETE2020, and FEDER through project PTDC/ASPAGR/30154/2017 (POCI-01-0145-FEDER-030154) is also acknowledged. The NMR spectrometer Bruker Avance III 400 (part of the National NMR Network) was financed by FCT and FEDER.

Acknowledgments: Thanks are due to Primor for the opportunity to perform the production of ham in a pilot scale, and to study this subject. Thanks are also due to Joel Borges and Filipe Vaz for the availability and prompt access to the colorimeter.

Conflicts of Interest: The authors declare no conflict of interest.

\section{References}

1. Sawicki, T.; Bączek, N.; Wiczkowski, W. Betalain profile, content and antioxidant capacity of red beetroot dependent on the genotype and root part. J. Funct. Foods 2016, 27, 249-261, doi:10.1016/j.jff.2016.09.004.

2. Nemzer, B.; Pietrzkowski, Z.; Spórna, A.; Stalica, P.; Thresher, W.; Michalowski, T.; Wybraniec, S. Betalainic and nutritional profiles of pigment-enriched red beet root (Beta vulgaris L.) dried extracts. Food Chem. 2011, 127, 42-53, doi:10.1016/j.foodchem.2010.12.081.

3. Chhikara, N.; Kushwaha, K.; Sharma, P.; Gat, Y.; Panghal, A. Bioactive compounds of beetroot and utilization in food processing industry: A critical review. Food Chem. 2019, 272, 192-200, doi:10.1016/j.foodchem.2018.08.022.

4. Ritz, T.; Werchan, C.A.; Kroll, J.L.; Rosenfield, D. Beetroot juice supplementation for the prevention of cold symptoms associated with stress: A proof-of-concept study. Physiol. Behav. 2019, 202, 45-51, doi:10.1016/j.physbeh.2019.01.015.

5. Silva, D.V.T.; Baião, D.S.; Silva, F.O.; Alves, G.; Perrone, D.; Aguila, E.M.D.; Paschoalin, V.M.F. Betanin, a natural food additive: stability, bioavailability, antioxidant and preservative ability assessments. Molecules 2019, 24, 458, doi:10.3390/molecules24030458.

6. Oliveira, G.V.; Morgado, M.; Pierucci, A.P.; Alvares, T.S. A single dose of a beetroot-based nutritional gel improves endothelial function in the elderly with cardiovascular risk factors. J. Funct. Foods 2016, 26, 301308, doi:10.1016/j.jff.2016.08.017.

7. Stintzing, F.C.; Herbach, K.M.; Mosshammer, M.R.; Carle, R.; Yi, W.; Sellappan, S.; Akoh, C.C.; Bunch, R.; Felker, P. Color, betalain pattern, and antioxidant properties of cactus pear (Opuntia stricta spp.) clones. J. Agric. Food Chem. 2005, 53, 442-451, doi:10.1021/jf048751y.

8. Silva, C.; Bolanho, B.C. Ultrasonic-assisted extraction of betalains from red beet (Beta vulgaris L.). J. Food Process. Eng. 2018, 41, 1-6, doi:10.1016/j.physbeh.2019.01.015. 
9. Zhang, H. Thin-film hydration followed by extrusion method for liposome preparation. Methods Mol. Biol. 2017, 1522, 17-22, doi:10.1007/978-1-4939-6591-5_2.

10. Jaafar-Maalej, C.; Diab, R.; Andrieu, V.; Elaissari, A.; Fessi, H. Ethanol injection method for hydrophilic and lipophilic drug-loaded liposome preparation. J. Liposome Res. 2010, 20, 228-243, doi:10.3109/08982100903347923.

(C) 2019 by the authors. Licensee MDPI, Basel, Switzerland. This article is an open access article distributed under the terms and conditions of the Creative Commons Attribution (CC BY) license (http://creativecommons.org/licenses/by/4.0/). 